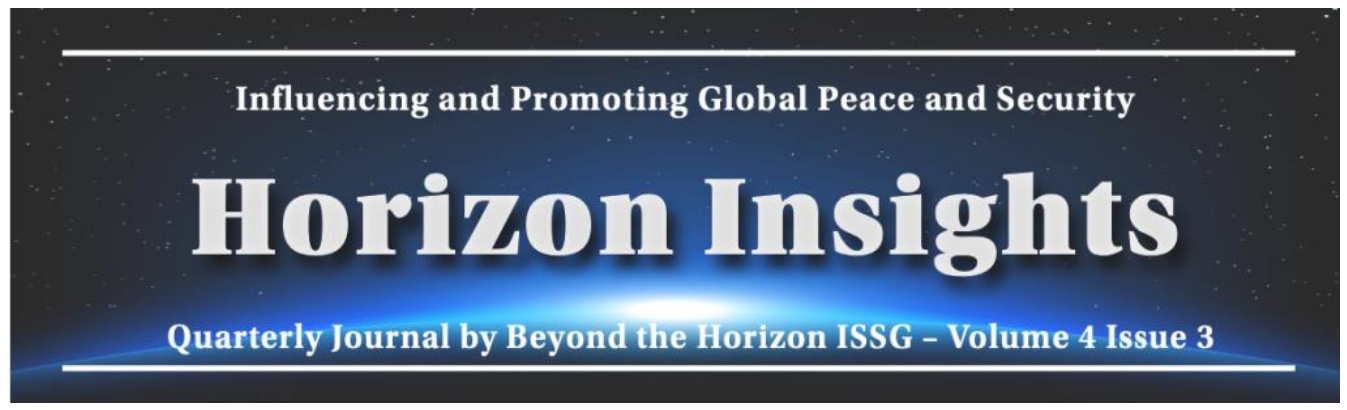

\title{
Book Review: Blockchain Revolution
}

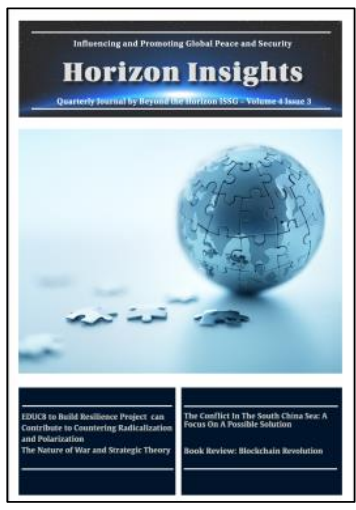

Tom Peeters

To cite this book review:

Peeters, T. (2021). Book Review: Blockchain Revolution [Review of the Book Blockchain Revolution, by D. Tapscott and A. Tapscott] Horizon Insights, 4 (3), 24-25. https://doi.org/10.31175/hi.2021.03.04

For the link to this issue of the journal:

https://behorizon.org/wp-content/uploads/2020/10/Horizon-Insights-2021-3.pdf

Horizon Insights Journal Homepage: https://behorizon.org/horizon-insights/

ISSN: 2593-3582 (printed), 2593-3590 (online)

Submit your article 
Tom Peeters ${ }^{1}$

UPDATED EDITION

With Material on Cryptoassets, ICOs, Smart Contracts, and More

BLOCKCHAIN REVOLUTION

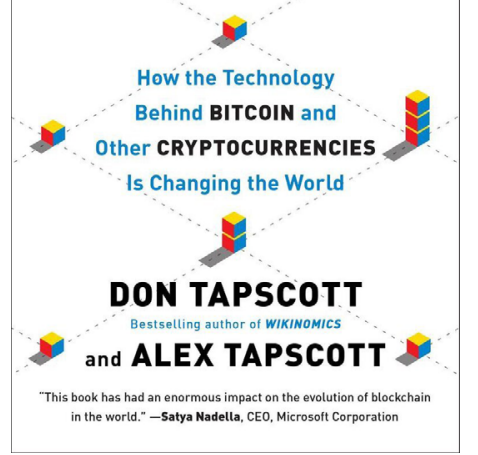

In 2008, the world was in financial turmoil. To solve this problem, a person named Satoshi Nakamoto came up with an idea that would soon spread like wildfire among computer scientists, banks, and anyone who had ever tried to establish trust on the Internet. We don't know who the person behind the name Satoshi Nakamoto is, honestly that doesn't matter anymore. What matters is his/her idea of virtual money that gave birth to "bitcoin" and "blockchain" technology. Blockchain is Cryptographically Secure. So, you might be wondering why it is called Blockchain. It's because every record, which could contain multiple transactions, is called a block on the ever-growing database. And each new block links to all previous blocks in a chain. This makes the system very secure and safe from tampering or hacking.

Special nodes called miners create new blocks. These contain new sets of transactions as well as links to the previous blocks and so on. When a new block is added, the miner who found it broadcasts that information to other nodes in the network. All those nodes check the

data and if they agree on what's in the block, then everything is fine.

In their book, Blockchain Revolution, the Authors Don and Alex Tapscott, co-founders of the Blockchain Research Institute, give information about the importance of Blockchain. The book explains how the power of this new technology behind Bitcoin can transform our world financially by improving the way we store our money and do business to make it more fair, transparent, equal, and free from corruption. Via Blockchain, you can eliminate corruption globally (however, there is still a long way to be successful).

The authors present their key points by explaining what blockchain technology is, how it can be used and why it has the potential to change the world. They also share their thought on the future of banking and how a few companies are making it obsolete. They explain why Airbnb and Uber aren't part of the sharing economy, as well as which is faster: sending an anvil to China or sending money through banks.

The book underscores the importance of identity and the end of digital feudalism. We saw what some called "surfing the Internet" as throwing off our data for the Internet landowners to expropriate and monetize. The notion of a self-sovereign identity for each of us, with our personal data stored in a virtual black box, is one of the most foundational concepts of our time. Realizing this "Virtual You" through blockchain technologies could restore our control over our own identities, the data we create, and the rest of our rights. As a thought experiment, the writers try to get inside Satoshi's mind and tease out his design principles for Blockchain. It turns out there were seven. After Chapter 2 which revolves around the technical background of the technology, they apply these seven principles to seven domains, namely financial services (chapter 3), the architecture of the firm (chapter 4), business model innovation (chapter 5), the Internet of Things (chapter 6), economic inclusion (chapter 7), government and democracy (chapter 8), and the creative industries (chapter 9)-and argue that Blockchain would create seven new substructures for a distributed economy.

The writers explain how Blockchain would radically reduce the transaction costs of search, coordination, contracting, and building trust in an open market. Inexorably, this efficiency will lead to more decentralized models for orchestrating the capabilities needed to create new products, services, and wealth.

It's almost impossible to cheat with the Blockchain; The proof-of-work algorithm is a way Bitcoin ensures that records are accurate without a central authority figure. Using Blockchain makes everything about

${ }^{1}$ Tom Peeters is a Non Resident Research Fellow at Beyond the Horizon ISSG 
finances quicker, cheaper, and more equal than our current banking system. Banks are unnecessarily slow and complicated. Via Blockchain, everything will be much more private and transparent, Blockchain users can choose their level of privacy by using cryptographic keys that can be their signature. Anyone can protect their privacy by choosing to use a new signature each time they make a transaction. But they can decide whatever level of privacy they want.

Blockchain is a system of verification that makes it impossible for hackers to make changes without being noticed. It also doesn't allow old entries to be changed since no one entity controls the Blockchain.

The Internet as we know it is excellent for collaboration and communication but is deeply flawed when it comes to commerce and privacy. The new blockchain technology facilitates peer-to-peer transactions without any intermediary such as a bank or governing body. The Blockchain validates and keeps a permanent public record of all transactions by keeping the user's information anonymous.

The Blockchain can hold any legal document, from deeds and marriage licenses to educational degrees and birth certificates. It enables smart contracts, decentralized autonomous organizations, decentralized government services, and transactions, among other things.

Blockchain solves the double-spend problem, as cryptographers call it. Now for the first time, we have a native digital medium for value, through which we can manage, store, and transfer any asset as money and music securely and privately.

The writers also discuss some implementation challenges/arguments in chapter 10 , like "inadequate incentives for distributed mass collaboration", "the blockchain is a job killer", "governing the protocols is like herding cats" and "criminals will use it". According to me, one of the most important challenges about Blockchain is "black money" and there is still a long way to tackle this issue.

Blockchain is the ingeniously simple technology that powers Bitcoin. But it is much more than that, too. It is a public ledger to which everyone has access but which no single person controls. It allows for companies and individuals to collaborate with an unprecedented degree of trust and transparency. It is cryptographically secure but fundamentally open. And soon, it will be everywhere.

The writers explain in detail about Blockchain, and you can find a comprehensive approach in the book, however, if you are not familiar with the Blockchain, it might be a little hard to understand precisely. I recommend everybody who would like to get in-depth information about Blockchain to read this book. This is a revised version of the groundbreaking book that came out in 2016. There is an entirely new preface to this edition of the book.

Don Tapscott is currently one of the world's leading authorities on the impact of technology on business and society having authored 16 widely read books and his son Alex Tapscott is following his father's path. It is crystal clear that Blockchain technology, especially crypto currencies (around $\$ 2,8 \mathrm{~T}$ market cap) will be permanent in our life in the future even though there are many questions and gaps about implementation for now. 